\title{
Comparison of PI controllers designed for the delay model of TCP/AQM networks
}

\author{
Hakkı Ulaş Ünal $^{\mathrm{a}, *}$, Daniel Melchor-Aguilar ${ }^{\mathrm{b}, 1}$, Deniz Üstebay ${ }^{\mathrm{c}}$, Silviu-Iulian Niculescu ${ }^{\mathrm{d}}$, Hitay Özbay ${ }^{\mathrm{e}}$ \\ ${ }^{a}$ Department of Electrical and Electronics Engineering, Anadolu University, 26555 Eskişehir, Turkey \\ ${ }^{\mathrm{b}}$ Division of Applied Mathematics, IPICyT, San Luis Potosi, SLP, Mexico \\ ${ }^{\mathrm{c}}$ Department of Electrical and Computer Engineering, McGill University, Montreal, Quebec, Canada \\ ${ }^{\mathrm{d}}$ Laboratoire des Signaux et Systèmes, CNRS-SUPELEC, 3 rue Joliot Curie, 91190 Gif-Sur-Yvette, France \\ ${ }^{\mathrm{e}}$ Department of Electrical and Electronics Engineering, Bilkent University, 06800 Ankara, Turkey
}

\section{A R T I C L E I N F O}

\section{Article history:}

Received 5 June 2012

Received in revised form 15 February 2013

Accepted 4 March 2013

Available online 16 March 2013

\section{Keywords:}

Active Queue Management

Delay systems

PI Controller

Network congestion

TCP/IP

\begin{abstract}
A B S T R A C T
One of the major problems of communication networks is congestion. In order to address this problem in $\mathrm{TCP} / \mathrm{IP}$ networks, Active Queue Management (AQM) scheme is recommended. AQM aims to minimize the congestion by regulating the average queue size at the routers. To improve upon AQM, recently, several feedback control approaches were proposed. Among these approaches, PI controllers are gaining attention because of their simplicity and ease of implementation. In this paper, by utilizing the fluid-flow model of TCP networks, we study the PI controllers designed for TCP/AQM. We compare these controllers by first analyzing their robustness and fragility. Then, we implement these controllers in ns-2 platform and conduct simulation experiments to compare their performances in terms of queue length. Taken together, our results provide a guideline for choosing a PI controller for AQM given specific performance requirements.
\end{abstract}

(c) 2013 Elsevier B.V. All rights reserved.

\section{Introduction}

Routers in a network transmit incoming packets to the destinations over links which have finite bandwidth. Thus, links can get congested if the amount of incoming packets exceeds the link capacity. When there are congested links in a network, the buffers of the routers might overflow and, consequently, new incoming packets might be lost. To address the congestion problem in TCP/ IP networks, queue management and scheduling algorithms are required at the routers. The traditional queue management technique at a router, known as tail drop, sets a maximum queue length in terms of packets and accepts packets for the queue until it overflows, then drops subsequent incoming packets until the queue decreases. Tail drop has some drawbacks such as flow synchronization, link under-utilization, and long end-to-end delay [1]. In order to overcome these drawbacks, Active Queue Management (AQM) scheme is recommended in [1]. The well-known AQM scheme is Random Early Detection (RED), which drops packets with

\footnotetext{
* Corresponding author. Tel.: +90 5353411964.

E-mail addresses: huunal@anadolu.edu.tr (H.U. Ünal), dmelchor@ipicyt.edu.mx (D. Melchor-Aguilar), deniz.ustebay@mail.mcgill.ca (D. Üstebay), Silviu.Niculescu@ lss.supelec.fr (S.-I. Niculescu), hitay@bilkent.edu.tr (H. Özbay).

1 Currently on a sabbatical leave in the Mechatronic Section, CINVESTAV-IPN, 07360 Mexico D.F., Mexico.
}

a probability that depends on the average queue length. Since RED drops packets by detecting the congestion, it significantly improves the link utilization compared to tail drop scheme. In addition, the flow-synchronization is eliminated and the effects of burst traffic are attenuated [2]. However, tuning RED parameters is a difficult task; if these parameters are not chosen carefully, the performance of RED can degrade, and, the system may become unstable. The stability of RED is investigated in $[3,4]$ by studying the maximum value of the packet marking probability that does not cause instability. As shown in [3], TCP/RED system becomes unstable if the round-trip delay and link capacity increase significantly, and/or the number of TCP sessions decreases drastically.

In order to obtain better performance compared to RED, by using the linearized fluid-flow model of TCP proposed in [5], several feedback control based advanced AQM controllers are proposed in the literature e.g., [6-9] and references therein. In [6], an $\mathcal{H}^{\infty}$ AQM controller was constructed by solving two-block $\mathcal{H}^{\infty}$ minimization problem to regulate the queue length against the variations of the plant parameters. By using the $\mu$-synthesis approach in [7], an $\mathcal{H}^{\infty} \mathrm{AQM}$ controller was designed considering delay-free part. In [8], by designing a robust observer, an $\mathcal{H}^{\infty}$ state feedback controller was designed to solve the same problem. In [9], an $\mathcal{H}^{\infty}$ state feedback was designed in order to solve the problem considering also the disturbances on the available bandwidth. However, it appears that these proposed controllers are not easy to 
implement in real networks due to their computational complexities.

In [10,11], a PI AQM controller design was proposed by using the small-gain theorem. It was shown there that PI controllers provide good responses in achieving AQM performance requirements. Based on this and their ease of implementation in real networks, several PI AQM controller designs have been proposed following those works, see for instance, [12-15]. Note that, from the practical implementation of a controller, it is required to keep the stability of the closed-loop system under round-off errors during implementation. A controller for which the closed-loop system can be destabilized by small perturbations in the controller coefficients is said to be fragile (see, e.g., [16]). There are only a few studies addressing the fragility problem of AQM controllers. To the best of the authors' knowledge, the first study was performed in [14], where a method to compute the largest available intervals for the PI controllers parameters have been developed. Recently in [15], using the complete characterization of the set of all stabilizing PI controllers and its corresponding geometric properties, a new method for tuning the parameters of PI/AQM controllers has been proposed. Such an approach allows us to design a PI controller stabilizing the network against perturbations in the network parameters. In addition, since the approach gives a simple procedure to determine the controller coefficients providing the maximum parametric stability margin in the controller's gains space, the designed PI controller stabilizes the network also against the perturbations on the coefficients of the controllers.

Although PI controllers are widely used in many control applications including complicated systems (see e.g., [17]), there does not exist a generally accepted tuning methodology. In addition, determining the PI parameters is a difficult task for many applications [18]. As pointed out in several surveys (see [18] and references therein), a high percentage of PI controllers have poor performances in many applications, due to bad controller tuning. Many tuning methods do not consider some restrictions such as unmodelled dynamics, non-linearities, and presence of delay. Another reason for the performance degradation of the PI-controllers is the uncertainties in the controller components due to the aging problem. This means that fragility of the controller should be taken into account

In this paper, we compare several PI controllers designed for TCP/AQM considering some performance requirements with arising problems in practice such as fragility and robustness. Some of these PI controllers are currently available for TCP/AQM given in $[11,15,14]$ and the other PI controllers are designed in the paper by utilizing the approaches in [19-23]. The designed PI controllers are based on considering the transfer function of the linearized model of TCP as an integrating system or a second order system with delay. In order to compare the robustness and fragility of the controllers, the stability region of all stabilizing PI AQM controllers for the considered network presented in [15] is utilized. For a performance comparison, the controllers are implemented in $n s-2^{2}$ and validated under different realistic scenarios considering various performance metrics.

It is worth mentioning that there also are propositions of PD and PID controllers for AQM schemes, see for instance, [25-28]. However, to the best of the authors' knowledge, the boundary of the stability region in the controller's parameters space of such controllers is not completely known and, therefore, an appropriate comparison of robustness and fragility issues can not be made as we performed here for PI AQM controllers.

\footnotetext{
2 ns-2 is a discrete event simulator that captures the stochastic and non-linear nature of the network dynamics [24]
}

The remainder of the paper is organized as follows. The mathematical model of the TCP fluid-flow model is given in Section 2. Various PI controller design methods for AQM schemes are summarized in Section 3. Section 4 provides a theoretical analysis of these PI controllers as well as simulation results comparing their performance in ns-2 platform. Concluding remarks are presented in Section 5.

\section{Mathematical model of the TCP flows}

In this section, we present the dynamical fluid-flow model developed by [11] for describing the behaviour of TCP/AQM networks. This model considers a network of $\mathrm{N}$ homogeneous TCPcontrolled sources and a single router. The average values of the key network variables are modelled by the following coupled and time-delayed non-linear differential equations:

$$
\begin{gathered}
\dot{W}(t)=\frac{1}{R(t)}-\frac{W(t)}{2} \frac{W(t-R(t))}{R(t-R(t))} p(t-R(t)), \\
\dot{q}(t)= \begin{cases}-C+\frac{N(t)}{R(t)} W(t), & q>0 \\
\max \left\{0,-C+\frac{N(t)}{R(t)} W(t)\right\}, & q=0\end{cases}
\end{gathered}
$$

where $W(t)$ is the average TCP window size (packets), $N(t)$ is the number of TCP sessions, $R(t)=\frac{q(t)}{C}+T_{o}$ is the round-trip time delay $(\mathrm{s}), q(t)$ is the average queue length (packets), $C$ is the link capacity (packets/s), $T_{o}$ is the propagation delay (s), and $p(t)$ is the probability of packet marking. Since the equations in (1) are non-linear, the transfer function for (1) can be obtained by making a linearization around their equilibrium points. In order to obtain the transfer function of (1), let $N(t)=N_{o}, C=C_{o}, W(t)=\delta_{W}(t)+W_{o}, q(t)=$ $\delta_{q}(t)+q_{o}$, and $p(t)=\delta_{p}(t)+p_{o}$, where $W_{o}, q_{o}, p_{o}$ are the equilibrium points determined by the nominal values. Then the transfer function from $\delta_{p}$ to $\delta_{q}$ can be obtained as in [11]:

$G_{p q}(s)=\frac{R_{0} C_{0} K}{\left(R_{0} s+\frac{1}{K}\right)\left(R_{0} s+1\right)} e^{-R_{0} s}$,

where $K=\frac{R_{0} C_{0}}{2 N_{0}}, R_{o}=T_{o}+\frac{q_{o}}{C_{0}}$. Therefore, by (2), it is possible to construct a closed-loop feedback system by designing PI controllers using various approaches, which are summarized in Section 3, for TCP/AQM model.

\section{PI controller design approaches for the delay model of TCP/ AQM}

In this section, several PI controller design approaches are summarized for the delay model of TCP/AQM. The stabilizing PI controllers are designed to provide a packet marking probability function as AQM strategy for regulating the average queue length at a desired operation point. Each PI controller has the structure

$K_{p i}(s)=K_{p}+\frac{K_{i}}{s}$,

where $K_{p}$ and $K_{i}$ correspond to the proportional and integral gains, respectively.

\subsection{PI controller design by Ziegler-Nichols approach}

Ziegler-Nichols approach is an empirical PID tuning method, which is based on the following steps:

- Set $K_{i}=0$. Stabilize the feedback system for a step reference $q_{0}$ with a very small gain $K_{p}$. 
- Gradually increase $K_{p}$ until output of the controller starts to oscillate. Then, record the gain $K_{p}$ as $K$ and oscillation period as $T$.

Then, the PI controller parameters are determined as $K_{p}=0.45 K$ and $K_{i}=\frac{K_{p}}{1.2} T$ [19].

\subsection{PI controller design by Panda et al. ([20])}

In this approach, PI controller design is presented for first order systems with time-delay considering the robustness by using the Internal Model Control (IMC) with Padé approximation. In order to design such a PI controller for AQM scheme, the approximation of the plant is obtained as

$G_{p q}(s) \approx \frac{R_{0} C_{0} K^{2}}{\tau_{m} s+1} e^{-D_{m} s}$,

where $\tau_{m}:=\left(0.828+\frac{0.812}{K}+0.172 R_{o} K e^{-\frac{6.9}{K}}\right)$ and $D_{m}:=1.116 \frac{R_{o} K}{K+1.208}+$ $R_{o}$. Then, the controller parameters are determined as $K_{p}=\frac{2 \tau_{m}+D_{m}}{2 R_{0} C_{0} K^{2} \lambda}$ and $K_{i}=\frac{1}{R_{0} C_{0} K^{2} \lambda}$, where $\lambda=\max \left\{\tau_{m}, 1.7 R_{o}\right\}$ (see also [29] for the details of the choice of $\lambda$ ).

\subsection{PI controller design by Hollot et al. ([11])}

This PI controller design is proposed to stabilize the feedback system with plant (2) against the high-frequency TCP parasitic. It is shown that the designed controller also stabilizes the system against the larger TCP sessions and smaller link capacity and round-trip time delay compared to nominal values, therefore, the resulting controller is robust. In this design method, the zero of PI controller is chosen to coincide with the corner frequency of the TCP window dynamic. Hence, if $L(s):=K_{p i}(s) G_{p q}(s)$ and $K_{p}=\frac{K_{i}}{z}$, then $z$ is chosen as $z=\frac{1}{R_{0} K}$. Therefore, the phase of the open-loop system depends on the TCP queue dynamics, and the round-trip time delay. In order to meet the crossover condition, i.e. $\left|L\left(j w_{g}\right)\right|=1, K_{i}$ is chosen as $K_{i}=w_{g} z\left|\frac{1+j R_{o} w_{g}}{C_{0} K}\right|$. Then, the phase of the open-loop transfer function can be written as

$\angle L\left(j w_{g}\right):=-90^{\circ}-\frac{180}{\pi} \beta-\arctan \beta$,

where $\beta:=w_{g} R_{0}$. Therefore, to design a stabilizing PI controller, $\beta$ should satisfy $\angle L\left(j w_{g}\right)-180^{\circ}>0$. Hence, once $w_{g}$ is chosen for design purposes, i.e. large bandwidth for a fast response, the stabilizing PI controller can be obtained provided that $\beta$ satisfies $\angle L\left(j w_{g}\right)-180^{\circ}>0$. Note that, large bandwidth requires larger $\beta$, which decreases the phase margin of the open-loop system, hence, deteriorates the system performance.

\subsection{PI controller design by Melchor-Aguilar, Niculescu ([15])}

In this approach, first the set of all robustly stabilizing PI controllers for the linearized model is determined. Then, by utilizing this set, a tuning methodology is presented to determine a non-fragile PI AQM controller. In order to design such a controller, let us introduce

$\sigma(t):=\int_{0}^{t}\left(q(v)-q_{0}\right) d v$

Then, by linearizing the augmented system (1)-(3) with the control law $\delta_{p}(t)=K_{p} \delta_{q}(t)+K_{i}\left(\sigma(t)-\frac{1}{K_{i}}\left(p_{o}-K_{p} q_{o}\right)\right)$, around the equilibrium points, it can be shown that the closed-loop system is exponentially stable if and only if

$f(s)=s^{3}+\frac{1}{R_{o}}\left(1+\frac{N_{o}}{R_{o} C_{o}}\right) s^{2}+\frac{2 N_{o}}{R_{o}^{3} C_{o}} s+\left[\frac{N_{o}}{R_{o}^{2} C_{o}} s^{2}+\frac{C_{o}^{2}}{2 N_{o}}\left(K_{p} s+K_{i}\right)\right] e^{-R_{o} s}$, has no zeros with non-negative real parts [30]. Following [15], the set of all robustly stabilizing PI controllers for the linearized model of TCP can be described as

$K_{p}(\omega)=\frac{2 N_{o}}{C_{o}^{2}}\left[\left(\omega^{2}-\frac{2 N_{o}}{R_{o}^{3} C_{o}}\right) \cos \left(\omega R_{o}\right)+\frac{\omega}{R_{o}}\left(1+\frac{N_{o}}{R_{o} C_{o}}\right) \sin \left(\omega R_{o}\right)\right]$,

$$
\begin{aligned}
K_{i}(\omega)= & \frac{2 N_{o} \omega}{C_{o}^{2}}\left[\frac{\omega}{R_{o}}\left(1+\frac{N_{o}}{R_{o} C_{o}}\right) \cos \left(\omega R_{o}\right)\right. \\
& \left.+\left(\frac{2 N_{o}}{R_{o}^{3} C_{o}}-\omega^{2}\right) \sin \left(\omega R_{o}\right)+\frac{N_{o} \omega}{R_{o}^{2} C_{o}}\right],
\end{aligned}
$$

where $w \in\left[\bar{w}, w^{*}\right]$. Here, $\bar{w}$ and $w^{*}$ are respectively the solution of

$$
\tan \left(\omega R_{o}\right)=\frac{\frac{2 N_{o}}{R_{0}^{3} C_{o}}-\omega^{2}}{\frac{\omega}{R_{o}}\left(1+\frac{N_{o}}{R_{o} C_{o}}\right)}
$$

and

$$
\frac{N_{o}}{R_{o}^{2} C_{o} \omega}=\frac{R_{o} \omega \sin \left(\omega R_{o}\right)-\cos \left(\omega R_{o}\right)}{R_{o} \omega\left(1+\cos \left(\omega R_{o}\right)\right)+2 \sin \left(\omega R_{o}\right)},
$$

where $w \in\left(0, \frac{\pi}{2 R_{0}}\right)$. Then, the stability region of all PI controllers for $\mathrm{TCP} / \mathrm{AQM}$ is determined by the coordinate axes $K_{p}=0$ and $K_{i}=0$ and the curve defined by (4) and (5). Now, once the stability region is obtained, in order to determine the non-fragile controller, the nominal controller parameters are chosen to put the largest circle in this region, where the radius of this circle represents the maximum $l_{2}$ parametric stability margin in the controller's gain space.

\subsection{PI controller design by Poulin, Pomerleau ([21])}

This approach is proposed for the integrating systems with time-delay. It is based on limiting the maximum peak-resonance $\left(\mathrm{M}_{r}\right)$ of the closed-loop transfer function to minimize the integral time of the absolute error (ITAE) due to the output step disturbance. In order to achieve this, the controller parameters are adjusted such that the transfer function of the open-loop system at the frequency where maximum phase occurs is tangent to the ellipse in the Nichols chart specified by the desired $M_{r}$ of the closed-loop system. To design such a PI controller for AQM, the considered plant has structure

$G_{p q}(s) \approx \frac{C_{0} K}{s\left(R_{0} s+1\right)} e^{-R_{o} s}$.

Note that this approximation is a well approximation of (1) if $K \gg 1$ [12]. Following [21], the PI parameters are chosen as $K_{p}=\frac{A_{\max }}{2 C_{0} K} \sqrt{\frac{R_{0}+2 T_{i}}{T_{i}^{2} R_{0}+2 R_{0}^{2} T_{i}}}, K_{i}=\frac{K_{p}}{T_{i}}, \quad$ where $\quad T_{i}=\frac{32 R_{o}}{\left(2 \phi_{\max }+\pi\right)^{2}}, \phi_{\max }=\arccos$ $\left(\frac{\sqrt{10^{0.1 \mathrm{M}_{\mathrm{r}}}-1}}{10^{0.05 \mathrm{M}_{\mathrm{r}}}}\right)-\pi, A_{\max }=\frac{10^{0.05 \mathrm{M}_{\mathrm{r}}}}{\sqrt{10^{0.1 \mathrm{M}_{\mathrm{r}}}-1}}$. The optimal $\mathrm{M}_{\mathrm{r}}$ values, which satisfy the design criterion, are plotted in Fig. 2 of [21] with respect to plant parameters. By utilizing that plot and considering (6), $M_{r}$ is chosen 4.25 .

\subsection{PI controller design by Üstebay, Özbay ([14])}

This approach is based on the work of [31] and aims to design a resilient controller in the sense of [32] for integrating systems with delay. In [31], firstly, the necessary and sufficient conditions for the stability of the closed-loop system are presented by utilizing the coprime factorizations of the considered plant and $K_{p i}$. Then, by using the small-gain theorem, the allowable intervals for $K_{p}$ and $K_{i}$ that ensure the stability of the closed-loop system are determined. To design a resilient PI controller for AQM, in [14], the transfer function in (6) is considered by assuming $K$ in (2) as $K \gg 1$. Then, it is shown 
that the optimal $K_{p}$, which maximizes the interval of allowable $K_{i}$, is found as $\frac{N_{o}}{2 R^{2} C_{0}^{2}}$. By the optimal $K_{p}$, the maximum value of the interval of allowable $K_{i}$ is found as $\frac{N_{o}}{16 R^{3} C^{2}}$. Then, to design a resilient controller, $K_{p}$ is chosen as $\frac{N_{o}}{2 R_{0}^{2} C_{0}^{2}}$ and $K_{i}$ is chosen as $\frac{N_{o}}{32 R_{0}^{3} C_{0}^{2}}$, which is the midpoint of the allowable interval for $K_{i}$.

\subsection{PI controller design by Wang, Shao ([22])}

In this method, the PI parameters are adjusted to minimize the integral error under a constraint such that the Nyquist curve of the open loop transfer function is tangent to a line parallel to the imaginary axis with a distance ensuring the stability margins. If $f\left(K_{p}, K_{i}, \omega\right):=\operatorname{Re}\left(K_{p i}(j \omega) G_{p q}(j \omega)\right)$, where $\operatorname{Re}(z)$ represents the real part of the complex number $z$, then, the constraint can be defined as

$f\left(K_{p}, K_{i}, \omega\right)=-\frac{1}{\lambda}$ with $\frac{\partial f\left(K_{p}, K_{i}, \omega\right)}{\partial \omega}=0$,

where $\lambda \in[1.5,2.5]$ for reasonable stability margins. Since the integral error is inversely proportional to $K_{i}$ [33], the controller parameters are obtained to maximize $K_{i}$ while satisfying (7). If we consider AQM problem, since $G_{p q}(j \omega)$ can be written as $G_{p q}(j \omega)=\alpha(\omega)+j \beta(\omega)$, where

$\alpha(\omega)=\frac{R_{o} C_{0} K}{n(\omega)}\left[\left(\frac{1}{K}-R_{o}^{2} \omega^{2}\right) \cos \left(R_{o} \omega\right)-\omega R_{o}\left(1+\frac{1}{K}\right) \sin \left(R_{o} \omega\right)\right]$,

$\beta(\omega)=\frac{-R_{0} C_{o} K}{n(\omega)}\left[\left(\frac{1}{K}-R_{o}^{2} \omega^{2}\right) \sin \left(R_{o} \omega\right)+\omega R_{o}\left(1+\frac{1}{K}\right) \sin \left(R_{o} \omega\right)\right]$,

$n(\omega)=\left(\frac{1}{K}-R_{o}^{2} \omega^{2}\right)^{2}+\left(\omega R_{o}+\frac{R_{o} \omega}{K}\right)^{2}$, then, the resulting controller parameters are obtained as

$K_{p}=\frac{1}{\left.\lambda \frac{d \alpha(\omega)}{d \omega}\right|_{\omega=\omega_{0}}}\left(\frac{1}{\beta\left(\omega_{0}\right)} \frac{d \beta(\omega)}{\left.d \omega\right|_{\omega=\omega_{0}}}-\frac{1}{\omega_{0}}\right)$

and $K_{i}=-\frac{w_{0}}{\lambda \beta\left(w_{0}\right)}$, where $\omega_{0}$ satisfying $\alpha\left(\omega_{0}\right)=0$ and $\lambda$ is chosen 2 , which is the midpoint of the interval for reasonable stability margins.

\subsection{PI controller design by Skogestad ([23])}

The PI controller design by this approach is based on two steps. In the first step, the original system is approximated to a first order system with delay. Since the delay term may limit the performance of the controller, an approximation technique, called "half rule", is recommended to reduce the conservativeness. Then, considering the system, obtained by "half rule", direct synthesis technique is used to provide the desired closed-loop system as a first order system with the same delay of the considered system. Since the resulting controller becomes "Smith Predictor", due to the direct synthesis technique and existence of delay in the desired response, Taylor series approximation is used to obtain a PI controller. In order to design a PI controller for AQM scheme, the first order approximation of (2) by "half rule" is obtained as

$G_{p q}(s) \approx \frac{R_{o} C_{0} K^{2}}{\left(K+\frac{1}{2}\right) R_{0} s+1} e^{-\frac{3}{2} R_{o} s}$.

Then, by using Skogestad-IMC settings, the controller parameters are obtained as

$K_{p}=\frac{1}{C_{o} K^{2}} \frac{K+1 / 2}{\tau_{c}+3 R_{o} / 2}$,

$K_{i}=\frac{K_{p}}{\min \left\{K R_{o}+R_{o} / 2,4\left(\tau_{c}+3 R_{o} / 2\right)\right\}}$, where $\tau_{c}$ is the time constant of the desired closed-loop response. For a fast response, good disturbance rejection and moderate robustness margins, $\tau_{c}=3 R_{o} / 2$ is recommended in [23].

\section{Comparison of the PI controllers}

In this section, we compare the designed controllers in the sense of fragility, robustness, and performance issues. The fragility and robustness properties of the controllers are compared using the stability region obtained by the approach of [15]. To validate and compare the performance issues of the controllers, we implement the controllers in ns- 2 and conduct simulations in different scenarios. Throughout the section, $\mathrm{PI}^{\mathrm{ZN}}, \mathrm{PI}^{\mathrm{PYH}}, \mathrm{PI}^{\mathrm{H}}, \mathrm{PI}^{\mathrm{MN}}, \mathrm{PI}^{\mathrm{PP}}, \mathrm{PI}^{\mathrm{UO}}$, $\mathrm{PI}^{\mathrm{WS}}$, and $\mathrm{PI}^{\mathrm{S}}$ correspond to the controller designed by the approach of Ziegler-Nichols, [20], [11,15], [21,14,22,23], respectively. For the sake of clarity, the PI controllers are designed for the same network parameters as in [11], i.e. $N_{o}=60, C_{o}=3750$ packets/s, and $R_{o}=0.246 \mathrm{~s}$. The corresponding proportional and integral gain values of each of the designed controller are given in Table 1 .

\subsection{Fragility and robustness comparisons}

The stability region of all stabilizing PI controllers for the considered network parameters is shown in Fig. 1. The region is determined by the coordinate axes $K_{p}=0, K_{i}=0$ and the curve defined by (4) and (5). As seen in Fig. 1, the designed controllers by each of the approaches belong to the stability region as expected.

In order to compare the fragility of each one of the designed controllers, let us define the following metric borrowed from [15]:

$\rho=\min \left\{K_{p}, K_{i}, \hat{\rho}\right\}$,

where $\hat{\rho}$ is the minimum distance from $\left(K_{p}, K_{i}\right)$ of each controller given in Table 1 to the boundary of the stability region computed by the approach of [15]. By (8), we get a circle with center at $\left(K_{p}, K_{i}\right)$ and radius $\rho$. Such a circle is the largest one inside the stability region that can be obtained for each of the designed controller's gains $\left(K_{p}, K_{i}\right)$, see Fig. 1 . Thus, a large $\rho$ yields a less fragile controller while a small $\rho$ leads to a more fragile controller. Hence, the controllers designed by $[11,14]$ are more fragile compared to the other controllers, as their $\rho$ values given in Table 2 are small. As seen in Table 2 and also shown in Fig. 1, controllers $\mathrm{PI}^{\mathrm{MN}}, \mathrm{PI}^{\mathrm{PYH}}, \mathrm{PI}^{\mathrm{ZN}}, \mathrm{PI}^{\mathrm{S}}$, and $\mathrm{PI}^{\mathrm{WS}}$ may not suffer fragility problem compared to the rest of the designed controllers. $\mathrm{PI}^{\mathrm{PYH}}$ is designed without taking into account the fragility issue, however, its distance to the boundary is close to the distance of $\mathrm{PI}^{\mathrm{MN}}$, which is the optimally non-fragile controller in the sense that it provides the greatest $l_{2}$ parametric margin.

For the robustness issue, we can compare the controllers in the sense of how much each of their parameters can be increased (with fixing the other one) without violating the stability. This issue is related to the classical gain margin problem. Therefore, let us define $\kappa_{i}\left(\kappa_{p}\right)$, which is the maximum gain such that $\kappa_{i} K_{i}\left(\kappa_{p} K_{p}\right)$ does not

Table 1

PI controller parameters.

\begin{tabular}{ll}
\hline \multicolumn{1}{c}{ Controllers } & $\left(K_{p}, K_{i}\right) \times 10^{-5}$ \\
\hline $\mathrm{PI}^{\mathrm{ZN}}$ & $(8.3745,11.375)$ \\
$\mathrm{PI}^{\mathrm{PYH}}$ & $(11.245,8.5981)$ \\
$\mathrm{PI}^{\mathrm{H}}$ & $(1.8182,0.9612)$ \\
$\mathrm{PI}^{\mathrm{MN}}$ & $(9.1044,6.8)$ \\
$\mathrm{PI}^{\mathrm{PP}}$ & $(3.7925,1.5987)$ \\
$\mathrm{PI}^{\mathrm{UO}}$ & $(3.5243,0.8953)$ \\
$\mathrm{PI}^{\mathrm{WS}}$ & $(4.1633,2.0146)$ \\
$\mathrm{PI}^{\mathrm{S}}$ & $(5.0046,2.4841)$ \\
\hline
\end{tabular}




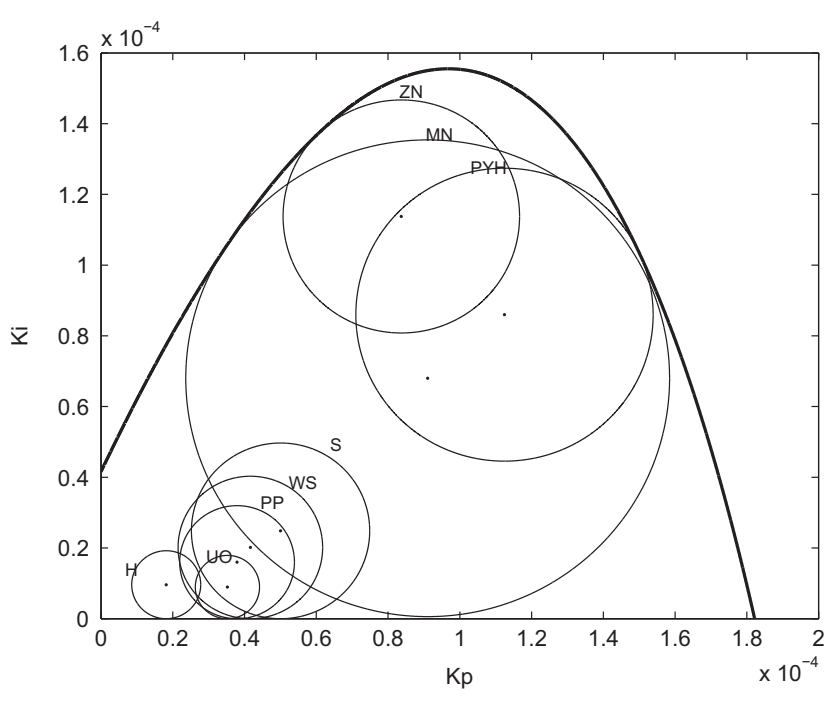

Fig. 1. Fragility comparison of the designed controllers.

Table 2

Fragility and robustness metrics of the controllers.

\begin{tabular}{llll}
\hline & $\rho \times 10^{-5}$ & $\kappa_{p}$ & $\kappa_{i}$ \\
\hline $\mathrm{PI}^{\mathrm{ZN}}$ & 3.2949 & 1.7314 & 1.3447 \\
$\mathrm{PI}^{\mathrm{PYH}}$ & 4.1421 & 1.3994 & 1.7630 \\
$\mathrm{PI}^{\mathrm{H}}$ & 0.9612 & 9.8983 & 8.0615 \\
$\mathrm{PI}^{\mathrm{MN}}$ & 6.7411 & 1.7988 & 2.2796 \\
$\mathrm{PI}^{\mathrm{PP}}$ & 1.5987 & 4.7057 & 6.8670 \\
$\mathrm{PI}^{\mathrm{UO}}$ & 0.8953 & 5.1114 & 11.819 \\
$\mathrm{PI}^{\mathrm{WS}}$ & 2.0146 & 4.2622 & 5.7095 \\
$\mathrm{PI}^{\mathrm{S}}$ & 2.4841 & 3.5221 & 5.0670 \\
\hline
\end{tabular}

destabilize the system with fixing the nominal value of $K_{p}\left(K_{i}\right)$. Clearly, in view of Fig. 1, in this case, a good choice would be to take a small nominal $K_{i}$ (respectively $K_{p}$ ). Performance constraint should determine the lower bounds for the nominal parameters. Note that $\kappa_{p}$ or $\kappa_{i}$ values for $\mathrm{PI}^{\mathrm{H}}, \mathrm{PI}^{\mathrm{UO}}$, and to some extent $\mathrm{PI}^{\mathrm{PP}}$ are larger compared to the corresponding values obtained with other controllers as presented in Table 2. So, these controllers are preferable vis-a-vis gain margin considerations.

\subsection{Performance comparisons}

For AQM, performance objectives include efficient queue utilization, low jitter, low packet dropping, and robustness with respect to varying network parameters. Now, we compare the performance of the PI controllers by implementing them in ns-2 considering different scenarios. The parameters of the PI controllers, given in Table 1, are obtained in the $s$ domain. However, for the implementation of these controllers in ns-2, each controller is converted to the $z$ domain by a sampling frequency chosen as 15 times of its open-loop bandwidth frequency [10].

For the simulations, we consider a dumbbell network given in Fig. 2. In the first 4 scenarios, the sources are TCP/Reno connections generating FTP flows, and in the last scenario, the sources generate UDP, HTTP and FTP flows. The capacity of the bottleneck is denoted by $C_{0}$ and the propagation delay between the routers is denoted by $T_{0}$. The pair $\left(C_{1}, T_{1}\right)$ represents the capacity of the links and the propagation delays between the sources and the first router. The pair $\left(C_{2}, T_{2}\right)$ represents the capacity of the links and the propagation delays between the second router and the sinks. In simulations, $q_{0}$ is taken as 200 packets with 400 packets buffer sizes for

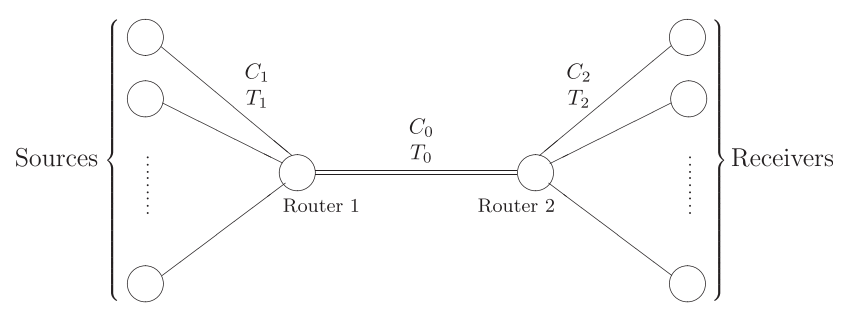

Fig. 2. Network topology.

each of the router, the average packet size is taken as 500 Bytes, and the simulation duration, $T_{\text {total }}$, is $200 \mathrm{~s}$.

In order to evaluate the performance of the designed PI controllers, we introduce five metrics related to the above performance objectives. The first metric is the RMS percentage error of the queue length with respect to the desired queue length $q_{0}$ :

$R M S_{\text {err }}=\left(\frac{1}{M} \sum_{i=1}^{M}\left(\frac{q(i)-q_{0}}{q_{0}}\right)^{2}\right)^{1 / 2}$,

where $M$ is the number of total samples generated by ns-2, $q(i)$ is the queue length at instant $i$. Note that since the buffer size is 400 packets, oscillation of $q(t)$ around 400 , i.e., hitting $q(t)$ to the buffer limit, implies the existence of congestion and packet dropping due to the saturation. Then, we can define the second metric as

$\Omega:=T_{s} / T_{\text {total }} \times 10^{3}$,

where $T_{s}$ is the total length of the time-intervals of $q(t)$ oscillating in the interval around 400, let us choose this interval as $[399,400]$. Here, $T_{s}$ can be thought of as the total time interval for buffer overflow, i.e. saturation, and packet dropping, hence, the controller which produces small $\Omega$ should be preferred. Note, since packet dropping may happen due to the larger overshoots, $\Omega$ does not give alone the complete packet loss. The link utilization is related with the time how long queue is efficiently used (i.e. the buffer is not empty) during the network traffic, hence, it is the function of total time intervals where $q(t) \neq 0$. Therefore, let $T_{z}$ be the total duration of the time when $q(t)$ drops to 0 . Since there will be no packet at the router during the time intervals lie in $T_{z}$, the link utilization can be defined as

$U:=$ utilization $=\frac{T_{\text {total }}-T_{z}}{T_{\text {total }}}$.

Then, by (9), $C_{u}:=(1-U) \times 10^{3}=\frac{T_{z}}{T_{\text {total }}} \times 10^{3}$ can be defined as the third metric. Since $T_{z}$ corresponds to the total duration of the link underutilized, the controller providing $U$ closest to 1 (or $C_{u}$ closest to 0 ) satisfies better link utilization compared to the other controllers. Now, let us define $\operatorname{Loss}_{r}$ as the ratio of the number of lost packets to the total number of sent packets by all the sources, then, we can define another metric as

$P_{\text {Loss }}=\operatorname{Loss}_{r} \times 10^{4}$.

Since minimization of the packet loss is one of the AQM performance objectives, the controller which provides small $P_{\text {Loss }}$ should be preferred. Another metric is related to the response speed of the controllers. We define this metric, called $R_{t}$, as the required time for $q(t)$ reaches $90 \%$ of the desired value but by discarding the time interval where $q(t)$ saturates the buffer capacity. In order to discuss jitter properties of the designed PI controllers, let us define

$R_{v}\left(t_{i}\right):=\frac{q\left(t_{i+1}\right)-q\left(t_{i}\right)}{t_{i+1}-t_{i}} \frac{1}{R_{0}} \times 10^{3}$,

where $q\left(t_{i}\right)$ is the queue length at discrete time $t_{i}$ generated by ns- 2 in the interval $\left(R_{t}, T_{\text {total }}\right)$. By the definition in (10), $R_{v}\left(t_{i}\right)$ can be considered as a relative delay variation at time $t_{i}$. 
Case 1: In this scenario, we consider the nominal response of the designed controllers. For this reason, the parameters of the network in the simulations are chosen as $N_{o}=60$ FTP flows, $C_{0}=C_{1}=C_{2}=15 \mathrm{Mbps}, T_{0}=192.7 \mathrm{~ms}, T_{1}=T_{2}=40 \mathrm{~ms}$. The performance analysis of the designed controllers are given in Table 3. As shown in Table 3, $\mathrm{PI}^{\mathrm{UO}}$ produces the smallest RMS error, while $\mathrm{PI}^{\mathrm{ZN}}$ produces the greatest RMS error compared to the other ones. Most of the controllers have the same link utilization performance, however, $\mathrm{PI}^{\mathrm{WS}}$ has the best one. Smallest packet dropping happens by $\mathrm{PI}^{\mathrm{MN}}$ and $\mathrm{PI}^{\mathrm{ZN}}$. Table 3 demonstrates that $\mathrm{PI}^{\mathrm{S}}$ and $\mathrm{PI}^{\mathrm{PYH}}$ have slower response than the other ones, while $\mathrm{PI}^{\mathrm{UO}}$ and $\mathrm{PI}^{\mathrm{PP}}$ have faster response. In order to compare the controllers which provide low jitter, by using (10), maximum $\left(\max _{R_{v}}\right)$, minimum $\left(\min _{R_{v}}\right)$, and average $\left(a v e_{R_{v}}\right.$ ) values of $\left\{R_{v}\left(t_{i}\right)\right\}_{t_{i} \in\left(R_{t}, T_{\text {tota }}\right)}$ for each of the designed PI controllers are presented in Table 4 . As seen in Table 4, the controllers $\mathrm{PI}^{\mathrm{UO}}, \mathrm{PI}^{\mathrm{PP}}$, and $\mathrm{PI}^{\mathrm{WS}}$ provide low jitter compared to other controllers. On the other hand, the controllers $\mathrm{PI}^{\mathrm{S}}$ and $\mathrm{PI}^{\mathrm{H}}$ result in large delay variations. As shown in Table 3, the controllers, which result in low jitter, provide small RMS error with high link utilization and the controllers, which result in large jitter, result in more packet dropping. The simulation results are presented in Figs. 3 and 4. As shown in all figures, the designed PI controllers regulate the queue length at the routers. Figs. 3(a) and 3(d) demonstrate that $\mathrm{PI}^{\mathrm{ZN}}$ makes large undershoots, whereas $\mathrm{PI}^{\mathrm{H}}$ makes large overshoots. The controllers $\mathrm{PI}^{\mathrm{WS}}$ and $\mathrm{PI}^{\mathrm{UO}}$, as seen in Fig. 3(b) and Fig. 4(c), result in small oscillations around the desired queue length and they have better steady-state responses. Note that, by considering Tables 3 and 4, the simulation results confirm that the controllers, which provide small queue oscillations around desired queue length, indicate low jitter, small RMS error, and also high link utilization.

Case 2: In this scenario, we consider the robustness property of the designed controllers. It has been shown in [15], by using geometric properties of the boundary of the stability region, that a stabilizing PI controller designed for network parameters $\left(N_{o}, C_{o}, R_{0}\right)$ also stabilizes a network with parameters $\left(\widetilde{N}_{o}, \widetilde{C}_{o}, \widetilde{R}_{o}\right)$, where $\widetilde{N}_{o} \geqslant N_{o}, \widetilde{C}_{o} \leqslant C_{o}$ and $\widetilde{R}_{o} \leqslant R_{o}$. Therefore, the number of FTP flows are taken 200 , link capacity at all the links are taken as $10 \mathrm{Mbps}$ and the propagation delay between routers is taken as

Table 3

Performance analysis of the PI controllers for Case 1.

\begin{tabular}{llllll}
\hline & $\mathrm{RMS}_{\text {err }}$ & $\Omega$ & $C_{u}$ & $\mathrm{P}_{\text {Loss }}$ & $R_{t}$ \\
\hline $\mathrm{PI}^{\mathrm{ZN}}$ & 0.4999 & 2.50 & 1.3520 & 3.2004 & 16.33 \\
$\mathrm{PI}^{\mathrm{WS}}$ & 0.4183 & 3.1344 & 0.8467 & 4.4172 & 14.62 \\
$\mathrm{PI}^{\mathrm{PYH}}$ & 0.4719 & 2.8725 & 1.3575 & 3.8865 & 17.59 \\
$\mathrm{PI}^{\mathrm{H}}$ & 0.4756 & 3.0517 & 0.8818 & 4.5030 & 14.30 \\
$\mathrm{PI}^{\mathrm{MN}}$ & 0.4557 & 2.4992 & 1.5114 & 3.1786 & 15.94 \\
$\mathrm{PI}^{\mathrm{PP}}$ & 0.4256 & 3.1288 & 0.8895 & 4.4806 & 13.71 \\
$\mathrm{PI}^{\mathrm{UO}}$ & 0.4091 & 3.1288 & 0.8895 & 4.4797 & 13.71 \\
$\mathrm{PI}^{\mathrm{S}}$ & 0.4693 & 3.2408 & 1.0319 & 5.3128 & 17.69
\end{tabular}

Table 4

Relative delay variation for Case 1

\begin{tabular}{lrll}
\hline & $\max _{R_{v}}$ & $\min _{R_{v}}$ & $a v e_{R_{v}}$ \\
\hline $\mathrm{PI}^{\mathrm{ZN}}$ & 13.548 & -8.129 & -1.9279 \\
$\mathrm{PI}^{\mathrm{PYH}}$ & 13.548 & -8.129 & -2.0260 \\
$\mathrm{PI}^{\mathrm{H}}$ & 20.322 & -8.129 & -1.9124 \\
$\mathrm{PI}^{\mathrm{MN}}$ & 13.548 & -8.129 & -1.9536 \\
$\mathrm{PI}^{\mathrm{PP}}$ & 8.129 & -8.129 & -1.8971 \\
$\mathrm{PI}^{\mathrm{UO}}$ & 8.129 & -8.129 & -1.8873 \\
$\mathrm{PI}^{\mathrm{WS}}$ & 8.129 & -8.129 & -1.9025 \\
$\mathrm{PI}^{\mathrm{S}}$ & 40.645 & -8.129 & -2.0656 \\
\hline
\end{tabular}

(a)

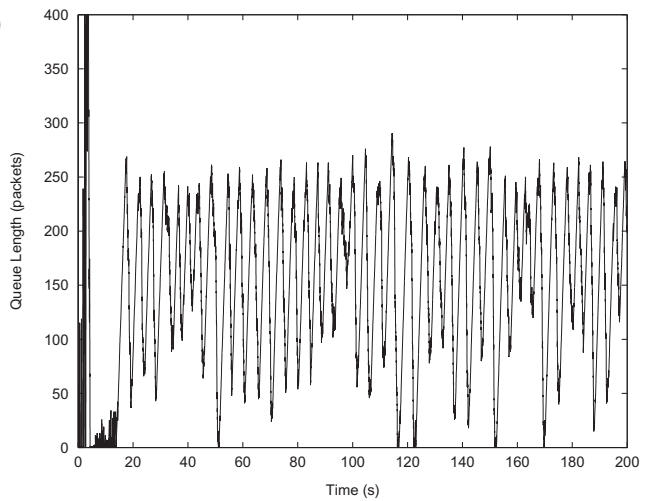

(b)

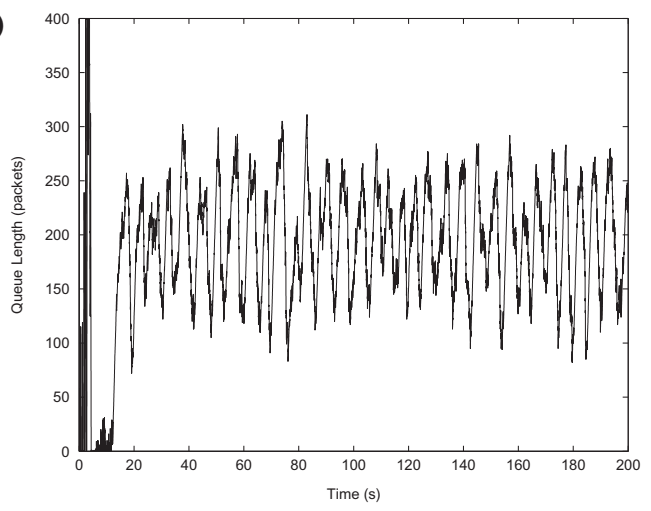

(c)

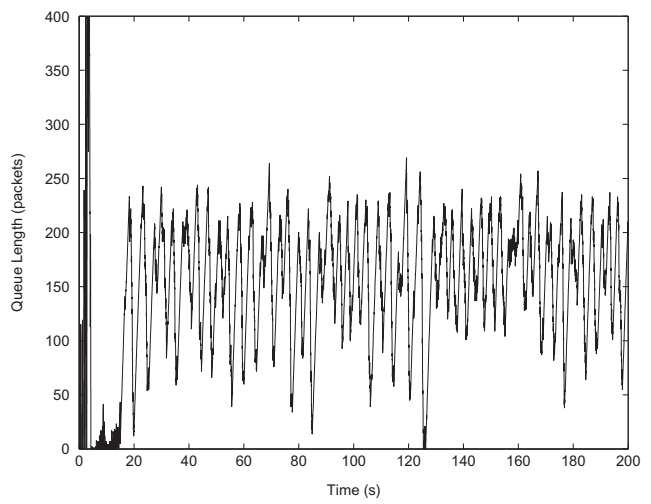

(d)

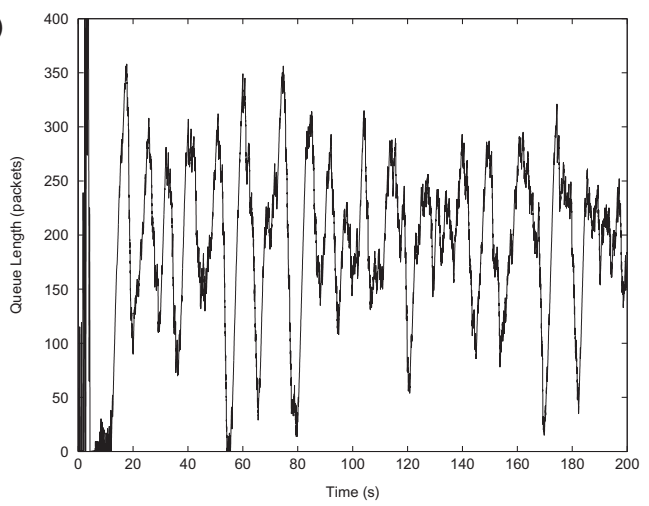

Fig. 3. Simulation results of (a) $\mathrm{PI}^{\mathrm{ZN}}$, (b) $\mathrm{PI}^{\mathrm{WS}}$, (c) $\mathrm{PI}^{\mathrm{PYH}}$ (d) $\mathrm{PI}^{\mathrm{H}}$ for Case 1 .

$T_{o}=43 \mathrm{~ms}$. The other simulation parameters are kept as in Case 1 . The performance analysis of the controllers are given in Table 5 . As seen in Table 5, the majority of the controllers provide less RMS error compared to Case 1, therefore, they regulate the queue length at the routers. In addition, compared to Case 1, the controllers pro- 
(a)

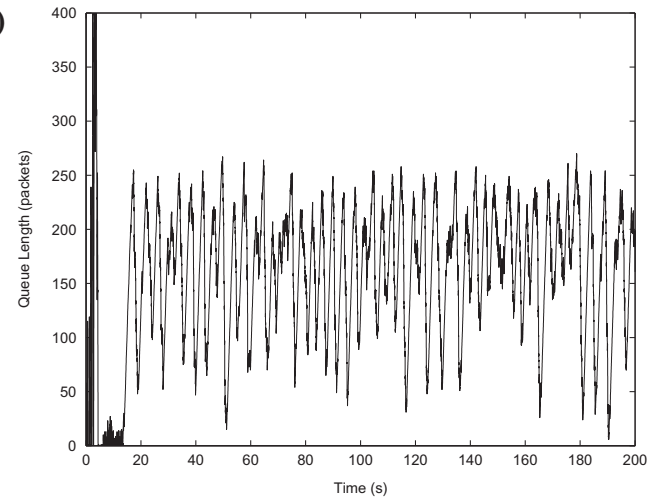

(b)

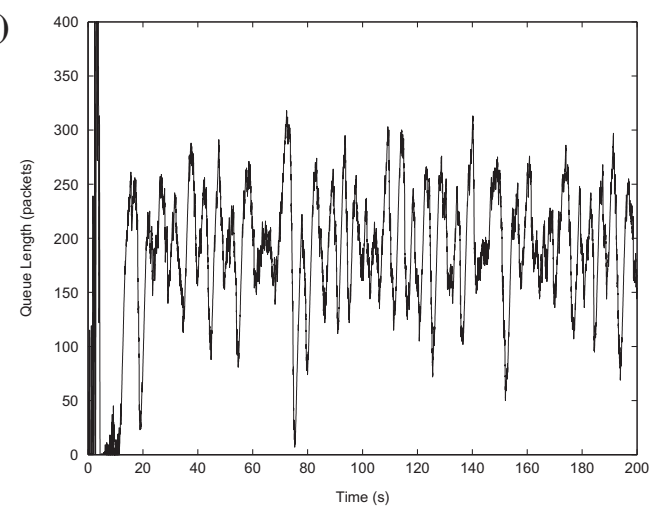

(c)

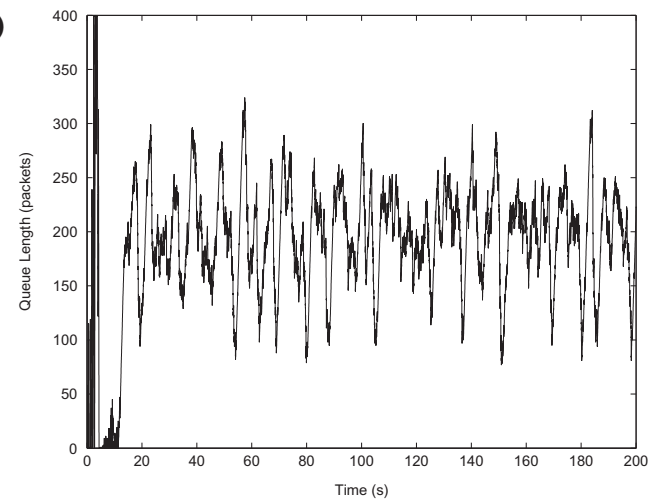

(d)

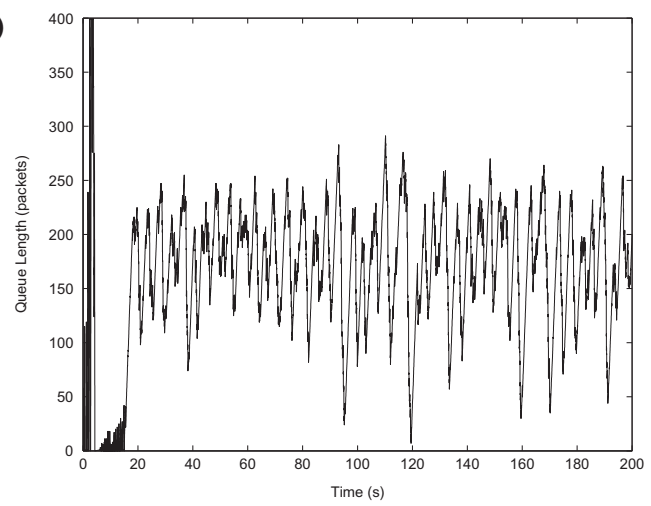

Fig. 4. Simulation results of (a) $\mathrm{PI}^{\mathrm{MN}}$, (b) $\mathrm{PI}^{\mathrm{PP}}$, (c) $\mathrm{PI}^{\mathrm{UO}}$ and (d) $\mathrm{PI}^{\mathrm{S}}$ for Case 1 .

vide better link utilization, however, more packets are dropped. One of the reasons for this result is the fact that the number of loads is taken more than 3 times of Case 1, hence, $q(t)$ oscillates around the upper limit of buffer for a long time as seen by comparing the second metric in Tables 3 and 5, therefore, more packet-
Table 5

Performance analysis of the PI controllers for Case 2.

\begin{tabular}{llllll}
\hline & $\mathrm{RMS}_{\text {err }}$ & $\Omega$ & $C_{u}$ & $\mathrm{P}_{\text {Loss }}$ & $\mathrm{R}_{\mathrm{t}}$ \\
\hline $\mathrm{PI}^{\mathrm{ZN}}$ & 0.2222 & 5.1938 & 0.1752 & 5.7922 & 14.85 \\
$\mathrm{PI}^{\mathrm{WS}}$ & 0.3553 & 13.214 & 0.1157 & 8.9505 & 62.58 \\
$\mathrm{PI}^{\mathrm{PYH}}$ & 0.2259 & 4.5065 & 0.1043 & 5.452 & 14.91 \\
$\mathrm{PI}^{\mathrm{H}}$ & 0.5134 & 35.186 & 0.1268 & 16.855 & 127.3 \\
$\mathrm{PI}^{\mathrm{MN}}$ & 0.2343 & 5.1727 & 0.1267 & 5.7779 & 20.96 \\
$\mathrm{PI}^{\mathrm{PP}}$ & 0.3908 & 16.078 & 0.1209 & 9.9564 & 62.82 \\
$\mathrm{PI}^{\mathrm{UO}}$ & 0.5170 & 27.738 & 0.0926 & 14.147 & 114.9 \\
$\mathrm{PI}^{\mathrm{S}}$ & 0.3284 & 11.662 & 0.1542 & 8.2289 & 43.38 \\
\hline
\end{tabular}

dropping happens. In addition, oscillation of $q(t)$ around its upper limit for a long period implies that $q(t)$ becomes zero only for a short time compared to Case 1 , hence, the link utilization is improved. From Table 5, $\mathrm{PI}^{\mathrm{H}}$ and $\mathrm{PI}^{\mathrm{UO}}$ yield larger RMS errors, $\mathrm{PI}^{\mathrm{ZN}}$, $\mathrm{PI}^{\mathrm{PYH}}$, and $\mathrm{PI}^{\mathrm{MN}}$ provide smaller RMS errors. The best link utilization is provided by $\mathrm{PI}^{\mathrm{UO}}$, and the rest of the controllers have similar levels of utilization. $\mathrm{PI}^{\mathrm{H}}$ and $\mathrm{PI}^{\mathrm{UO}}$ yield more packet dropping than the others, while $\mathrm{PI}^{\mathrm{PYH}}, \mathrm{PI}^{\mathrm{MN}}$ and $\mathrm{PI}^{\mathrm{ZN}}$ provide relatively small packet dropping. The last column of Table 5 shows that $\mathrm{PI}^{\mathrm{H}}$ and $\mathrm{PI}^{\mathrm{UO}}$ have slower response, while $\mathrm{PI}^{\mathrm{ZN}}, \mathrm{PI}^{\mathrm{PYH}}, \mathrm{PI}^{\mathrm{MN}}$ have faster response. As discussed above, $\mathrm{PI}^{\mathrm{UO}}$, which provides the best link utilization, and $\mathrm{PI}^{\mathrm{H}}$ yield large RMS error due to the fact that they saturate for a long time as shown by the second metric in Table 5. However, such a long saturation duration results in slower response and the controllers provide small oscillations around the desired queue length. Hence, these controllers result in low jitter compared to other controllers.

Case 3: In this scenario, we aim to evaluate the response of the controllers for a large nominal plant gain. The number of FTP flows is 45 , link capacities $C_{0}, C_{1}$ and $C_{2}$ are $18 \mathrm{Mbps}$ and the propagation delay between the routers is set to $T_{o}=350 \mathrm{~ms}$. The rest of the simulation parameters are kept as in Case 1 . The performance analysis of the controllers are given in Table 6. As shown by the table, performances of all the controllers are deteriorated, they result in larger RMS error and worse link utilization compared to the previous scenarios. In addition, since the controllers yield $q(t)$ to become zero frequently due to the worse link utilization, as seen in Table 6, fewer packets are dropped compared to Cases 1 and 2. As seen from Table 6 , most of the controllers produce the same RMS error. Among these controllers, $\mathrm{PI}^{\mathrm{H}}, \mathrm{PI}^{\mathrm{PP}}, \mathrm{PI}^{\mathrm{UO}}$, and $\mathrm{PI}^{\mathrm{WS}}$ result in the smaller RMS error, $\mathrm{PI}^{\mathrm{ZN}}$ and $\mathrm{PI}^{\mathrm{MN}}$ result in the larger RMS error and worse link utilization, while $\mathrm{PI}^{\mathrm{S}}$ and $\mathrm{PI}^{\mathrm{H}}$ provide the better link utilization. Most of the controllers yield the same packet dropping, however, $\mathrm{PI}^{\mathrm{PYH}}$ yields the minimum packet dropping, $\mathrm{PI}^{\mathrm{WS}}$, $\mathrm{PI}^{\mathrm{PP}}$, and $\mathrm{PI}^{\mathrm{UO}}$ result in larger packet droppings. The response time of the most of the controllers are close to each other, however, $\mathrm{PI}^{\mathrm{MN}}$ is the controller which has a slowest response, while $\mathrm{PI}^{\mathrm{H}}$ and $\mathrm{PI}^{\mathrm{PYH}}$ have faster response. Since the controllers yield worse link utilization with larger RMS error, they have worse performance in the sense of jitter compared to the previous cases.

Case 4: In this scenario, the gain of the nominal plant is larger than the one in Case 3. The number of FTP flows are taken 30, link capacities $C_{0}, C_{1}$ and $C_{2}$ are taken $18 \mathrm{Mbps}$ and the propagation delay between the routers is taken as $T_{0}=537.6 \mathrm{~ms}$. The other simulation parameters are kept as in Case 1. As seen by the performance analysis of the controllers given in Table 7, the controllers have worse performances in the sense of RMS error but better performance in the sense of lost packet ratio compared to the previous cases. Additionally, all the controllers, except $\mathrm{PI}^{\mathrm{ZN}}$ and $\mathrm{PI}^{\mathrm{PYH}}$, have better link utilization compared to Case 3. From Table 7 , the maximum RMS error is produced by $\mathrm{PI}^{\mathrm{ZN}}$, and the other controllers produce RMS errors close to each other. The better link 
Table 6

Performance analysis of the PI controllers for Case 3.

\begin{tabular}{llllll}
\hline & RMS $_{\text {err }}$ & $\Omega$ & $C_{u}$ & $\mathrm{P}_{\text {Loss }}$ & $\mathrm{R}_{\mathrm{t}}$ \\
\hline $\mathrm{PI}^{\mathrm{ZN}}$ & 0.7188 & 1.7703 & 11.056 & 3.2220 & 47.89 \\
$\mathrm{PI}^{\mathrm{WS}}$ & 0.6153 & 1.9390 & 5.5956 & 3.6808 & 44.88 \\
$\mathrm{PI}^{\mathrm{PYH}}$ & 0.6700 & 0.9952 & 6.9587 & 1.8312 & 41.58 \\
$\mathrm{PI}^{\mathrm{H}}$ & 0.5482 & 1.8281 & 3.9471 & 3.3795 & 41.21 \\
$\mathrm{PI}^{\mathrm{MN}}$ & 0.7153 & 1.7703 & 11.079 & 3.2234 & 50.40 \\
$\mathrm{PI}^{\mathrm{PP}}$ & 0.6085 & 1.9390 & 5.8341 & 3.6749 & 43.89 \\
$\mathrm{PI}^{\mathrm{UO}}$ & 0.6272 & 1.9390 & 6.0446 & 3.6673 & 47.25 \\
$\mathrm{PI}^{\mathrm{S}}$ & 0.6550 & 1.7957 & 3.8808 & 3.2332 & 44.32
\end{tabular}

Table 7

Performance analysis of the PI controllers for Case 4.

\begin{tabular}{llllll}
\hline & RMS $_{\text {err }}$ & $\Omega$ & $C_{u}$ & $\mathrm{P}_{\text {Loss }}$ & $\mathrm{R}_{\mathrm{t}}$ \\
\hline $\mathrm{PI}^{\mathrm{ZN}}$ & 0.8341 & 0.8967 & 25.51 & 1.9321 & 115.1 \\
$\mathrm{PI}^{\mathrm{WS}}$ & 0.7610 & 1.2249 & 3.1337 & 2.3703 & 116.7 \\
$\mathrm{PI}^{\mathrm{PYH}}$ & 0.8137 & 0.9014 & 8.1792 & 1.7468 & 124.8 \\
$\mathrm{PI}^{\mathrm{H}}$ & 0.7787 & 1.6062 & 1.4446 & 3.6100 & 129.1 \\
$\mathrm{PI}^{\mathrm{MN}}$ & 0.7857 & 0.8967 & 9.9103 & 1.8741 & 115.1 \\
$\mathrm{PI}^{\mathrm{PP}}$ & 0.7767 & 1.2249 & 4.5427 & 2.3750 & 116.7 \\
$\mathrm{PI}^{\mathrm{UO}}$ & 0.7751 & 1.2249 & 2.3877 & 2.3695 & 116.7 \\
$\mathrm{PI}^{\mathrm{S}}$ & 0.7713 & 1.1117 & 2.9463 & 2.2004 & 125.0 \\
\hline
\end{tabular}

Table 8

Performance analysis of the PI controllers for Case 5.

\begin{tabular}{llllll}
\hline & RMS $_{\text {err }}$ & $\Omega$ & $C_{u}$ & $\mathrm{P}_{\text {Loss }}$ & $\mathrm{R}_{\mathrm{t}}$ \\
\hline $\mathrm{PI}^{\mathrm{ZN}}$ & 0.3758 & 2.7466 & 1.4798 & 5.4678 & 9.08 \\
$\mathrm{PI}^{\mathrm{WS}}$ & 0.3126 & 2.5742 & 0.8874 & 5.1014 & 8.22 \\
$\mathrm{PI}^{\mathrm{PYH}}$ & 0.3479 & 2.4980 & 1.3401 & 4.8442 & 8.54 \\
$\mathrm{PI}^{\mathrm{H}}$ & 0.3597 & 3.8994 & 0.7907 & 6.8847 & 7.95 \\
$\mathrm{PI}^{\mathrm{MN}}$ & 0.3408 & 2.6530 & 0.8956 & 5.2050 & 9.15 \\
$\mathrm{PI}^{\mathrm{PP}}$ & 0.3123 & 2.5644 & 0.8330 & 5.0743 & 8.45 \\
$\mathrm{PI}^{\mathrm{UO}}$ & 0.3212 & 2.5644 & 1.0573 & 5.0645 & 8.44 \\
$\mathrm{PI}^{\mathrm{S}}$ & 0.3170 & 2.8621 & 1.2066 & 5.5632 & 8.64 \\
\hline
\end{tabular}

utilization is provided by $\mathrm{PI}^{\mathrm{H}}$, which causes more packet dropping. $\mathrm{PI}^{\mathrm{PYH}}, \mathrm{PI}^{\mathrm{ZN}}$ and $\mathrm{PI}^{\mathrm{MN}}$ provide small packet droppings. The last column of Table 7 shows that the controllers have slower response compared to the ones in previous cases. Among the controllers, $\mathrm{PI}^{\mathrm{H}}$ is the slowest one. Longer response time and worst link utilization can be attributed to the drastic increase in the open-loop gain. As discussed in Case 3, the controllers in this case may result in high jitter compared to the previous cases.

Case 5: We here consider a more realistic traffic scenario. The network sources, link capacity and propagation delay between the routers change dynamically. We consider 180 HTTP sessions (180 clients and 1 server), 60 FTP flows, and 10 UDP flows with a packet size 250 bytes. Therefore, $75 \%$ of the traffic consists of short-lived flows, called web mice, which make the traffic more realistic [34]. The UDP flows follow an exponential ON/OFF traffic model such that both the idle and burst times have mean of $0.5 \mathrm{~ms}$ and the sending rate during the on-time is $0.05 \mathrm{Mbps}$. The propagation delay of each UDP flow uniformly varies within the interval $[20,80] \mathrm{ms}$ and these flows are active between $t=50 \mathrm{~s}$ and $t=150 \mathrm{~s}$. We introduce dynamic load $N_{o}(t)$ such that at $t=80 \mathrm{~s}, 30$ of the FTP flows drop out and at $t=140 \mathrm{~s}$ they return. The propagation delay $T_{o}$ and the link capacity $C_{0}$ uniformly vary within the interval $[100,300] \mathrm{ms}$ and $[12,18]$ Mbps respectively. The rest of the simulation parameters are kept as in Case 1. The (a)

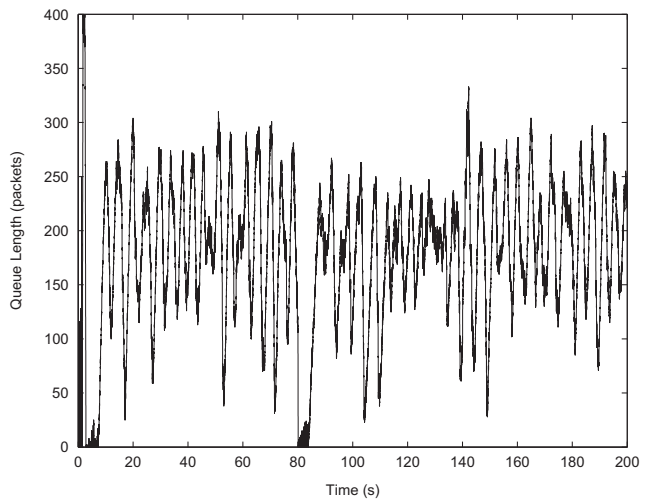

(b)

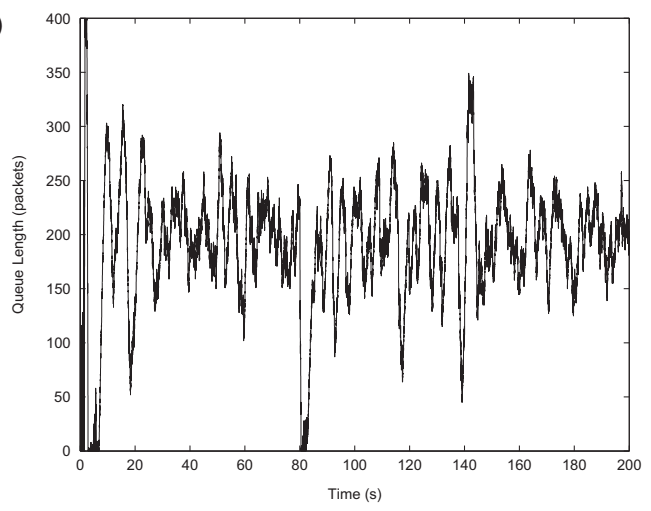

(c)

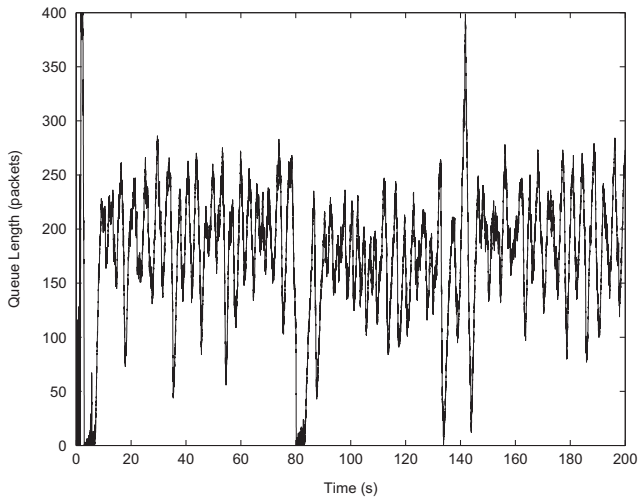

(d)

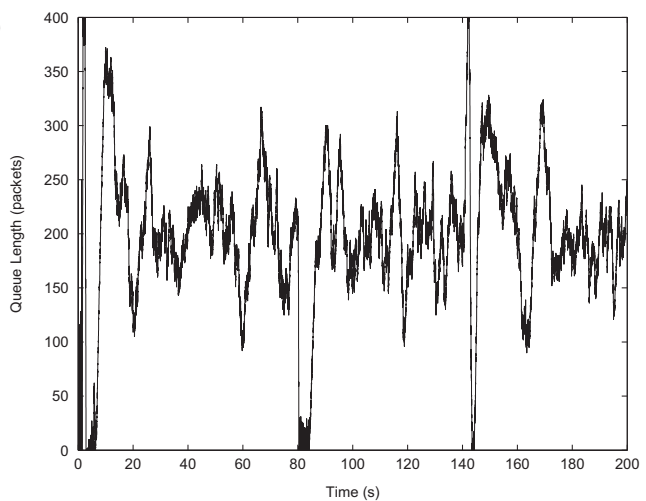

Fig. 5. Simulation results of (a) $\mathrm{PI}^{\mathrm{ZN}}$, (b) $\mathrm{PI}^{\mathrm{WS}}$, (c) $\mathrm{PI}^{\mathrm{PYH}}$ (d) $\mathrm{PI}^{\mathrm{H}}$ for Case 5 .

performance analysis of the controllers are given in Table 8. As seen from the table, $\mathrm{PI}^{\mathrm{PP}}$ and $\mathrm{PI}^{\mathrm{WS}}$ provide small RMS error, while $\mathrm{PI}^{\mathrm{ZN}}$ provides the largest one. The link utilization performance of most of the controllers are close to each other, however, $\mathrm{PI}^{\mathrm{H}}$ is the best one and $\mathrm{PI}^{\mathrm{ZN}}$ is the worst. $\mathrm{PI}^{\mathrm{H}}$ yields more packet dropping, 
(a)

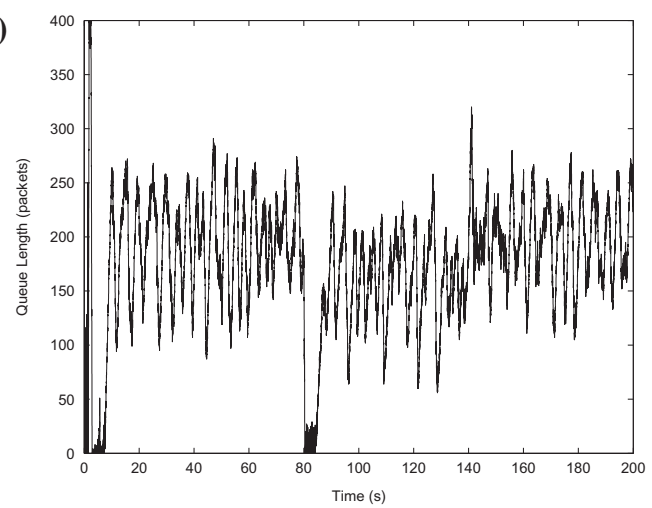

(b)

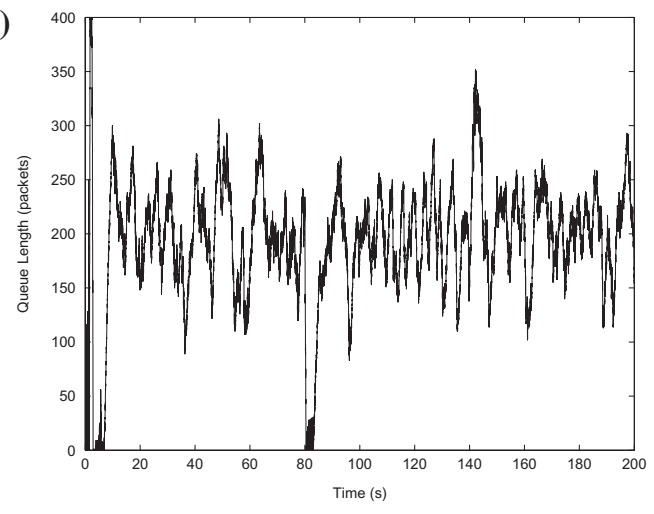

(c)

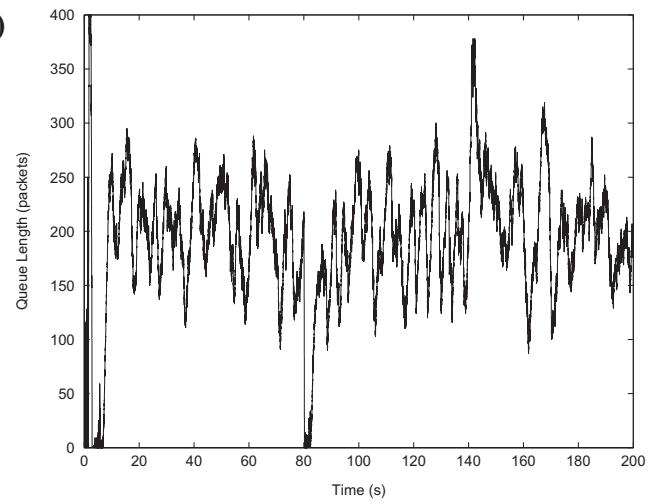

(d)

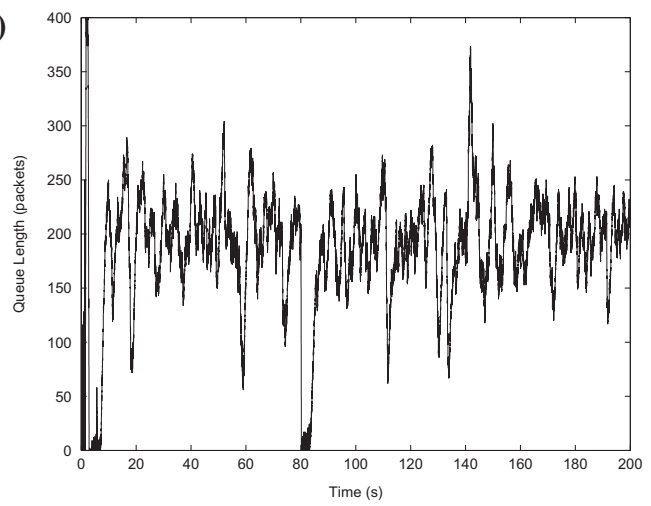

Fig. 6. Simulation results of (a) $\mathrm{PI}^{\mathrm{MN}}$, (b) $\mathrm{PI}^{\mathrm{PP}}$, (c) $\mathrm{PI}^{\mathrm{UO}}$ and (d) $\mathrm{PI}^{\mathrm{S}}$ for Case 5 .

whereas $\mathrm{PI}^{\mathrm{PYH}}$ provides less packet dropping. The response time of controllers are close each other, however, $\mathrm{PI}^{\mathrm{H}}$ has fast response compared to the others, while $\mathrm{PI}^{\mathrm{MN}}$ has slowest response. The simulation results are presented in Figs. 5 and 6. As seen from the figures, when the number of sources drop to 30 , at $t=80 \mathrm{~s}$, the queue length drops to 0 suddenly and the link is underutilized for a short duration. As seen in Fig. 5(b), PI ${ }^{\mathrm{WS}}$ acts faster compared to the other controllers to regulate the queue length due to the change of the number of sources. When these sources return at $t=140 \mathrm{~s}$, as seen in Fig. 5(d), for the controller $\mathrm{PI}^{\mathrm{H}}$, queue length drops to 0 for some duration compared to other controllers, which means that the link is underutilized. In addition, as seen in Fig. 5(c), 5(d) and Figs. 6(c), 6(d), $\mathrm{PI}^{\mathrm{PYH}}, \mathrm{PI}^{\mathrm{H}}, \mathrm{PI}^{\mathrm{UO}}$, and $\mathrm{PI}^{\mathrm{S}}$ have aggressive responses and larger overshoots. As seen in Fig. $5(\mathrm{~d}), \mathrm{PI}^{\mathrm{H}}$ result in larger overshoots around the desired queue length, which may result in larger jitter. Similarly, $\mathrm{PI}^{\mathrm{ZN}}$, which provide largest RMS error and worst link utilization, may also result in large jitter. Since queue length in Fig. 5(b), and Fig. 6(b) does not make large overshoots around its desired level, the controllers $\mathrm{PI}^{\mathrm{WS}}$ and $\mathrm{PI}^{\mathrm{PP}}$, which provide small RMS error with high link utilization, may also provide low jitter. As seen in Fig. 5(a), $\mathrm{PI}^{\mathrm{ZN}}$ is less robust to unresponsive flows, since the controller results in large undershoots when UDP sources switched on and off.

\subsection{Discussions}

As shown in Figs. 3-6, the designed controllers regulate the queue length at the desired level. For large TCP sessions, as in Case 2 , the designed controllers still regulate the queue length. However, if the nominal plant gain is drastically increased, then, the controllers loose their ability to regulate the queue length. As seen in Case 5, the designed PI AQM controllers achieve the objectives on the queue length in a dynamic traffic scenario and in presence of disturbance factors such as short-lived and UDP flows.

To recap, the least fragile PI controllers are $\mathrm{PI}^{\mathrm{MN}}$ and $\mathrm{PI}^{\mathrm{PYH}}$. In the sense of maximizing $\kappa_{p}$ (respectively $\kappa_{i}$ ) the best PI controllers are $\mathrm{PI}^{\mathrm{H}}$ (respectively $\mathrm{PIO}^{\mathrm{UO}}$ ). Since $\mathrm{PI}^{\mathrm{PP}}$ is designed to bound the $\mathrm{M}_{\mathrm{r}}$ of the closed-loop transfer function, it bounds the complementary sensitivity function, so it has some robustness property. In general, the controller $\mathrm{PI}^{\mathrm{ZN}}$ gives the largest RMS error, and, $\mathrm{PI}^{\mathrm{MN}}$ and $\mathrm{PI}^{\mathrm{PYH}}$, provide the smaller packet dropping. In most of the cases studied, $\mathrm{PI}^{\mathrm{ZN}}$ and $\mathrm{PI}^{\mathrm{MN}}$ have worst link utilization, while $\mathrm{PI}^{\mathrm{UO}}$ and $\mathrm{PI}^{\mathrm{H}}$ have the best link utilization. Considering the response time of the controllers, $\mathrm{PI}^{\mathrm{ZN}}$ has faster response in most cases. In general, $\mathrm{PI}^{\mathrm{WS}}, \mathrm{PI}^{\mathrm{PP}}$, and $\mathrm{PI}^{\mathrm{UO}}$ provide low jitter, while $\mathrm{PI}^{\mathrm{ZN}}$ and $\mathrm{PI}^{\mathrm{H}}$ result in high jitter. Note that, additional analysis and simulation results for different scenarios are presented in [35].

\section{Conclusion}

In this paper, a comparison of various PI controllers designed for the delay-model of TCP/AQM is performed. The compared PI controllers are the ones currently available for TCP/AQM proposed in $[11,14,15]$ and the designed ones for TCP/AQM in the current paper using the approaches given in [19-23]. It should be noted that, it is possible to find different PI controller design approaches for TCP/ AQM than the presented ones in the paper. However, we believe that the chosen PI controller design approaches are more appropriate considering the linear model of TCP/AQM among the others in the literature. The comparison in the paper is based on the fragility, robustness, and performance issues of the controllers. For a comprehensive and realistic comparison, we implemented the controllers in ns-2 for different traffic scenarios and validated the results considering some AQM performance objectives.

Our analysis showed that the PI controllers of $[15,20]$ are less fragile compared to the controllers designed by the approach of Zeigler-Nichols, and $[22,11,21,14,23]$. On the other hand, the controllers proposed by $[11,14,21,22]$ are more robust compared with the rest because their proportional or integral gain margins are relatively large. Additionally we note that the controller designed by 
the methods of [21,11] have also good robustness property in the sense that they limit the sensitivity function. The simulation results indicate that, in general, robust controllers have better link utilization and provide low jitter, while the controllers, which may not suffer "fragility" problem, provide smaller packet dropping.

Note that the approaches of [14,21] are for the simplified model of the system $K \gg 1$, which may result in conservativeness. In addition, since the approach of [14] is based on the small-gain theorem, like [11], another conservativeness arises. The controllers proposed by $[20,23]$ can also be considered conservative because they are designed using a first order approximation of the transfer function. The approach of [15], however, gives the set of all stabilizing controllers, and allows us to measure the parametric stability margins.

In conclusion, we have identified which PI controllers to choose for different performance and robustness metrics in AQM. As in all other application areas, these controllers form a baseline for an initial design; depending on the performance requirements of a particular network, PI controllers can be modified for further improvements.

\section{Acknowledgments}

The authors thank the Associate Editor and reviewers for their useful suggestions that helped us to improve the paper. This work is supported in part by the French-Turkish PIA Bosphorus (TUBITAK Grant No. 109E127 and EGIDE Project No. 22974WJ). The work of Hakkı Ulaş Ünal is supported by the project STRT1-09/33 of the K.U. Leuven Research Council. The work of Hitay Özbay is partially supported by DPT-HAMIT project. The work of Daniel MelchorAguilar is supported by CONACYT Grant 131587.

\section{References}

[1] B. Braden, D. Clark, J. Crowcroft, B. Davie, S. Deering, D. Estrin, S. Floyd, V. Jacobson, G. Minshall, C. Partridge, L. Peterson, K. Ramakrishnan, S. Shenker, J. Wroclawski, L. Zhang, Recommendations on queue management and congestion avoidance in the Internet, RFC 2309.

[2] S. Ryu, C. Rump, C. Qiao, Advances in Internet congestion control, IEEE Communications Surveys and Tutorials 5 (1) (2003) 28-39.

[3] L. Tan, W. Zhang, G. Peng, G. Chen, Stability of TCP/RED systems in AQM routers, IEEE Transactions on Automatic Control 51 (8) (2006) 1393-1398.

[4] W. Zhang, L. Tan, G. Peng, Dynamic queue level control of TCP/RED systems in AOM routers, Computers and Electrical Engineering 35 (1) (2009) 59-70.

[5] V. Misra, W.B. Gong, D. Towsley, Fluid-based analysis of a network of AQM routers supporting TCP flows with an application to RED, in: Proceedings of ACM/SIGCOMM, Stockholm, Sweden, 2000, pp. 151-160.

[6] P.F. Quet, H. Özbay, On the design of AQM supporting TCP flows using robust control theory, IEEE Transactions on Automatic Control 49 (2004) 1031-1036.

[7] Q. Chen, Q.W.W. Yang, Robust controller design for AQM router, IEEE Transactions on Automatic Control 52 (2007) 938-943.

[8] S. Manfredi, M. Bernardo, F. Garofalo, Design, validation and experimental testing of a robust AQM control, Control Engineering Practice 17 (2009) 394 407.
[9] F. Zheng, J. Nelson, An $\mathcal{H}_{\infty}$ approach to the controller design of AQM routers supporting TCP flows, Automatica 45 (3) (2009) 757-763.

[10] C.V. Hollot, V. Misra, D. Towsley, W. Gong, On designing improved controllers for AQM routers supporting TCP flows, in: Proceedings of IEEE INFOCOM Alaska, USA, 2001, pp. 1726-1734.

[11] C.V. Hollot, V. Misra, D. Towsley, W. Gong, Analysis and design of controllers for AQM routers supporting TCP flows, IEEE Transactions on Automatic Control 47 (6) (2002) 945-959.

[12] W. Michiels, D. Melchor-Aguilar, S.-I. Niculescu, Stability analysis of some classes of TCP/AQM networks, International Journal of Control 79 (2006) 1136-1144.

[13] D. Melchor-Aguilar, V. Castillo-Tores, Stability analysis of proportional-integral AQM controllers supporting TCP flows, Computacion y Sistemas 10 (2007) 401-414.

[14] D. Üstebay, H. Özbay, Switching resilient PI controllers for active queue management of TCP flows, in: Proceedings of the 2007 IEEE International Conference on Networking, Sensing and Control, London, 2007, pp. 574-578.

[15] D. Melchor-Aguilar, S.-I. Niculescu, Computing non-fragile PI controllers for delay models of TCP/AQM networks, International Journal of Control 82 (2009) 2249-2259.

[16] L.H. Keel, S.P. Bhattacharyya, Robust, fragile, or optimal?, IEEE Transactions on Automatic Control 42 (8) (1997) 1098-1105

[17] W. Michiels, S.I. Niculescu, Stability and Stabilization of Time-Delay Systems: An Eigenvalue-Based Approach, SIAM, Philadelphia, PA, 2007.

[18] C.-C. Yu, Autotuning of PID Controllers: A Relay Feedback Approach, Springer, Germany, 2006.

[19] Q.C. Zhong, Robust Control of Time-Delay Systems, Springer-Verlag, London, UK, 2006.

[20] R.C. Panda, C.-C. Yu, H.P. Huang, PID tuning rules for SOPDT systems: review and some new results, ISA Transactions 43 (2004) 283-295.

[21] E. Poulin, A. Pomerleau, PID tuning for integrating and unstable processes, Control Theory and Applications, IEE Proceedings 143 (1996) 429-435.

[22] Y.-G. Wang, H.-H. Shao, Optimal tuning for PI controller, Automatica 36 (2000) 147-152.

[23] S. Skogestad, Simple analytic rules for model reduction and PID controlle tuning, Journal of Process Control 13 (2003) 291-309.

[24] The network simulator, ns-2 [online], 2000. <http://www.isi.edu/nsnam/ns/>

[25] J. Sun, K.-T. Ko, G. Chen, S. Chan, M. Zukerman, PD-RED: to improve the performance of RED, IEEE Communications Letters 7 (8) (2003) 406-408.

26] X. Deng, S. Yi, G. Kesidis, C.R. Das, A control theoretic approach for designing adaptive AQM schemes, in: IEEE Global Telecommunications Conference (GLOBECOM 2003) USA, San Francisco, 2003, 2947-2951.

[27] S. Ryu, C. Rump, C. Qiao, Advances in active queue management (AQM) based TCP congestion control, Telecommunication Systems 25 (2004) 317-351.

[28] D. Üstebay, H. Özbay, N. Gündeş, A new PI and PID control design method for integrating systems with time delays, in: Proceedings of the 6th WSEAS International Conference on Signal Processing, Robotics and Automation, Corfu Island, Greece, 2007, pp. 60-65.

[29] A. O'Dwyer, Handbook of PI and PID Controller Tuning Rules, Imperial College Press, London, 2006

[30] J.K. Hale, S.M.V. Lunel, Introduction to Functional Differential Equations, Applied Mathematics Science, vol. 99, Springer-Verlag, New York, 1993.

[31] H. Özbay, A.N. Gündeş, Resilient PI and PD controller designs for a class of unstable pants with I/O delays, Applied and Computational Mathematics 6 (2007) 18-26.

[32] G.J. Silva, A. Datta, S. Bhattacharyya, PID Controllers for Time-Delay Systems, Birkhäuser-Boston, USA, 2004

[33] K.J.Âström, T. Hägglund, PID controllers: Theory, Design and Tunning, Instrument Society of America, Research Triangle Park, NC, 1995.

[34] M. Christiansen, K. Jeffay, D. Ott, F.D. Smith, Tuning RED for Web traffic, IEEE ACM Transactions on Networking 9 (3) (2001) 249-264.

[35] H.U. Ünal, D. Melchor-Aguilar, D. Üstebay, S.I. Niculescu, H. Özbay, Comparing PI controllers for delay models of TCP/AQM networks, in: Proceedings of the 4th IFAC Symposium on Systems, Structure, and Control, Ancona, Italy, 2010 pp. 76-83. 